\title{
IDENTIFICAÇÃO DE DIFICULDADES DE INTERAÇÃO NA GESTÃO DE DESIGN PARA CONCEPÇÃO DE UMA FERRAMENTA DE APOIO À GESTÃO DE DESIGN: UMA ABORDAGEM SISTÊMICA
}

\author{
IDENTIFICATION OF INTERACTION DIFFICULTIES IN DESIGN \\ MANAGEMENT FOR CREATING A TOOL TO DESIGN MANAGEMENT: \\ A SYSTEMIC APPROACH
}

\author{
SILVA, Carina Scandolara da (1); \\ MERINO, Eugenio Díaz (2); \\ MERINO, Giselle Schmidt Alvez Díaz (3); \\ FIGUEIREDO, Luiz Fernando Gonçalves de (4) \\ (1) Universidade Federal de Santa Catarina, Mestre \\ e-mail: cariscan@gmail.com \\ (2) Universidade Federal de Santa Catarina, Doutor \\ e-mail: eugenio.merino@ufsc.br \\ (3) Universidade Federal de Santa Catarina, Doutora \\ e-mail: gisellemerino@gmail.com \\ (4) Universidade Federal de Santa Catarina, Doutor \\ e-mail: Iff@cce.ufsc.br
}

\begin{abstract}
RESUMO
Projetar produtos buscando usabilidade é proporcionar que usuários alcancem seus objetivos e satisfaçam suas necessidades. A pesquisa realizada teve como objetivo levantar as principais dificuldades e necessidade de interação entre componentes da gestão de design, por meio de uma abordagem sistêmica, afim de gerar informação que possa colaborar para o estabelecimento de requisitos para o desenvolvimento de uma ferramenta de suporte à gestão de design. Isso se deu por meio de uma estudo de caso, num estúdio de design, e também pela continuidade de um trabalho realizado no mesmo contexto. Por meio da pesquisa realizada, identificou-se as principais dificuldades encontradas na interação entre componentes da gestão de design e que a ferramenta pode facilitar 0 controle e o acesso às informações concernentes aos projetos, objetivos e estratégias.
\end{abstract}

Palavras-chave: Usabilidade; Interação; Gestão de Design; Abordagem Sistêmica

\section{ABSTRACT}

Search usability in product design is to provide users achieve their goals and meet their needs. The research aims to identify the main difficulties and needs in design management interaction components, 
through a systematic approach in order to generate information that can contribute to the establishment of conditions for the development of a support tool in design management. This is achieved through a case study, a design studio, and also the continuity of research already carried out in the same context. Through the survey, we identified the main difficulties encountered in the interaction between design and management components that the tool can facilitate the control and access to information concerning the plans, objectives and strategies.

Keywords : Usability, Interaction, Design Management, System Approach

\section{INTRODUÇÃO}

Projetar e avaliar computadores buscando usabilidade, conforme a NBR ISO 9241-11 (2002), é proporcionar que usuários alcancem seus objetivos e satisfaçam suas necessidades em um contexto particular de uso. A usabilidade planejada para um produto pode ser definida, documentada e verificada. Também poder ser melhorada pela incorporação de características e atributos capazes de beneficiar os usuários em um contexto particular de uso.

A temática da pesquisa desenvolvida refere-se ao levantamento de informações relativas ao contexto de uso para o desenvolvimento de uma ferramenta de suporte à gestão de design. Tem o objetivo geral de levantar as principais dificuldades e necessidade de interação entre componentes da gestão de design, por meio de uma abordagem sistêmica, afim de gerar informação que possa colaborar para o estabelecimento de requisitos para o desenvolvimento de uma ferramenta de suporte à gestão de design.

A pesquisa se justifica pela necessidade de desenvolver produtos adequados aos usuários deste sua concepção, levando-se em conta não apenas as suas funcionalidades e desempenho, mas também o conforto e a satisfação dos usuários. A necessidade de uma ferramenta de apoio à gestão de design foi identificada em um estudo elaborado por Silva (2012), que estudava a abordagem sistêmica com foco na gestão de design sustentável. Um dos resultados da pesquisa foi um mapeamento sistêmico do funcionamento da gestão de design de um estúdio de design de pequenos porte, em que consta as interações entre os componentes da gestão de design naquele ambiente. Este resultado foi empregado no desenvolvimento desta pesquisa, que realizou-se no mesmo estúdio de design da anterior, e levantou as necessidades em relação à ferramenta que se deseja desenvolver e as principais dificuldades que são encontradas nas interações entre os componentes da gestão de design. Por meio da pesquisa realizada, foi identificado que a ferramenta de suporte à gestão de design deveria facilitar o controle e o acesso às informações concernentes aos projetos, objetivos e estratégias da gestão de design, bem como tentar melhorar as dificuldades encontradas no contexto da gestão de design.

\section{USABILIDADE NA CONCEPÇÃO DE PRODUTOS}

A usabilidade surgiu no contexto de ergonomia de interface humano-computador e, ao longo dos últimos anos vem evoluindo para considerar os produtos de forma geral (CYBIS ET AL, 2010). É definida como sendo a "medida na qual um produto pode ser usado por usuários específicos para alcançar objetivos específicos com eficácia, eficiência e satisfação em um contexto específico de uso." (NBR 9241-11, 2002: 3) 
Para o melhor compreensão desta definição, é importante conhecer o significado dos seguintes termos, de acordo com Jordan (1998):

- Eficácia: se refere à extensão na qual uma meta é alcançada ou uma tarefa é realizada.

- Eficiência: se refere à quantidade de esforço requerido para se atingir uma meta. Quanto menos esforço, maior é a eficiência.

- Satisfação: se refere ao nível de conforto que os usuários sentem quando utilizam um produto e também ao nível de aceitação do produto pelos usuários para atingir as suas metas.

A "usabilidade é uma abordagem para o desenvolvimento de produtos que incorpora, de forma direta, o feedback do usuário ao longo do seu ciclo de desenvolvimento de forma a reduzir custos e a criar produtos e ferramentas que atendam as necessidades dos usuários." (Associação dos Profissionais de Usabilidade - UPA, 2013). Diversos autores fazem a definição de usabilidade, cada um com sua especificidade. Porém, o que se pode deduzir é que buscam um resultado que atenda às necessidades dos usuários, sustentando que é possível planejar o resultado pretendido.

De acordo com a NBR ISO 9241-11 (2002:4), "planejar para usabilidade, como parte de um projeto e desenvolvimento de produtos, envolve uma identificação sistemática de requisitos para usabilidade, incluindo medidas de usabilidade e descrições verificáveis do contexto de uso. Estas fornecem metas de projeto que podem servir de base para a verificação do projeto resultante." A norma também organiza uma estrutura para especificar a usabilidade que orienta como descrever a proposta do produto, os componentes de usabilidade, os objetivos, 0 contexto de uso e as medidas de usabilidade.

Figura 1: Estrutura de usabilidade NBR ISO

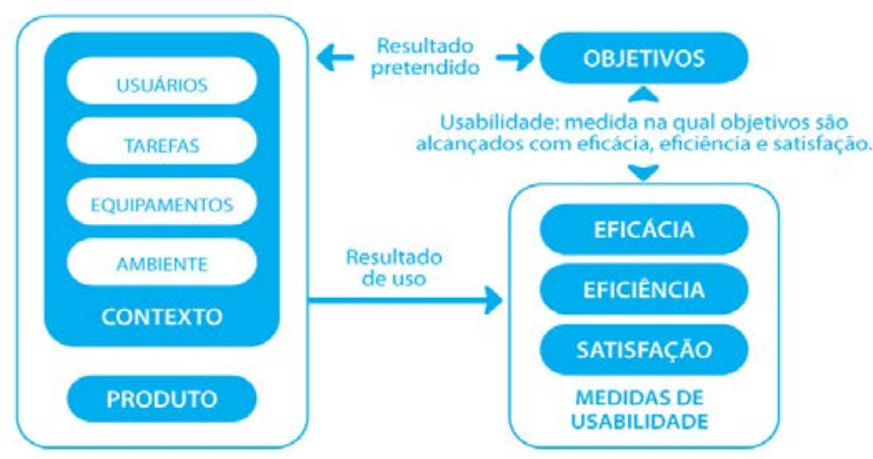

Fonte: NBR ISO 9241-11 (2002:4)

A figura 1 mostra o diagrama com a estrutura de usabilidade sugerida pela NBR ISO 9241-11 (2002), mostrando o ciclo que o produto deve atender. Na primeira coluna, constam os itens que devem ser descritos e que vão fornecer as informações necessárias para desenvolver um produto segundo seu objetivo pretendido e que tenha eficácia, eficiência e proporcione satisfação ao usuário. 
Para o levantamento das informações sobre o contexto da gestão de design foi adotada uma abordagem sistêmica, com o uso de mapeamento sistêmico e ferramentas auxiliares para coleta de dados e informações sobre o contexto. Estas informações darão suporte para posterior elaboração de uma ferramenta de apoio à gestão de design.

\section{ABORDAGEM SISTÊMICA DA GESTÃO DE DESIGN}

A visualização de situações sob uma abordagem sistêmica vem sendo feita em diversas áreas como gestão, processos de engenharia, computação, gestão ambiental etc. A Teoria Geral dos Sistemas - TGS, de Bertalanffy (2008), e as teorias de autores que buscam o pensamento sistêmico como forma de abordagem, falam sobre "uma ciência geral da 'totalidade',[...] mas aplicável às várias ciências empíricas", (BERTALANFFY, 2008:62). Foi daí que se deu o aparecimento, nos campos da ciência, de noções tais como totalidade, holístico, organísmico, gestalt etc., significando que se deve pensar em sistemas de elementos em interação mútua.

Bertalanffy (2008) defende que um sistema ou uma complexidade organizada pode ser definido pela existência de fortes interações ou de interações não triviais, ou seja, não lineares. Fala que é necessário estudar não somente as partes e processos isoladamente, mas também resolver os problemas decisivos encontrados na organização e na ordem que os unifica, resultante da interação dinâmica das partes, tornando o comportamento das partes diferente quando estudado isoladamente e quando tratado no todo.

$\mathrm{Na}$ visão de Capra (1993), a concepção sistêmica entende o mundo em termos de relações e de integração. Os sistemas são totalidades integradas, cujas propriedades não podem ser reduzidas às de unidades menores e que todo e qualquer organismo é uma totalidade integrada e, portanto, um sistema vivo. Mas os sistemas não estão limitados a organismos individuais e suas partes, pois os mesmos aspectos de totalidade são exibidos por sistemas sociais. Embora se possa discernir partes individuais em qualquer sistema, a natureza do todo é sempre diferente da mera soma de suas partes.

$\mathrm{Na}$ pesquisa realizada, buscou-se voltar para a gestão de design este olhar sistêmico e abrangente com foco nas relações entre as diversas partes do sistema, tendo também um panorama do todo, afim de compreender o problema de forma sistêmica. Sistematizar a gestão de design foi a forma encontrada para executar prática sistêmica, que se caracteriza pela aplicação do pensamento sistêmico de forma concreta. (Vasconcelos, 2001)

A Gestão de Design caracteriza-se por um "conjunto de técnicas de gestão empresarial dirigidas a maximizar, ao menor custo possível, a competitividade que a empresa obtém pela incorporação e utilização do design industrial como instrumento de sua estratégia organizacional" (GIMENO, 2000, p. 25). E tem como papel "a implantação do design na empresa para ajudar a construir sua estratégia, o que implica na: i) gestão de integração do design na empresa em nível operacional do projeto, organizacional da função ou estratégico da missão; e ii) na gestão do sistema de design da empresa". (MOZOTA, 2002, p.86)

A gestão de design é dividida por Best (2009) e Mozota (2003) em três níveis: o estratégico, o tático e o operacional. Sendo que: 
- O nível estratégico, ou a habilidade de design para unificar e transformar a visão da organização, define as diretrizes, as missões e o planejamento;

- O nível tático ou funcional, ou a criação de uma função de design na organização, é representado pelas equipes, pelo processo e pelas funções específicas necessárias;

- O nível operacional de projeto, ou o primeiro passo para o design integrado, se manifesta nos produtos e serviços que o consumidor vai poder se apropriar.

Figura 2: Componentes do sistemas em cada nível de gestão de design para Best (2009) e Mozota (2003)

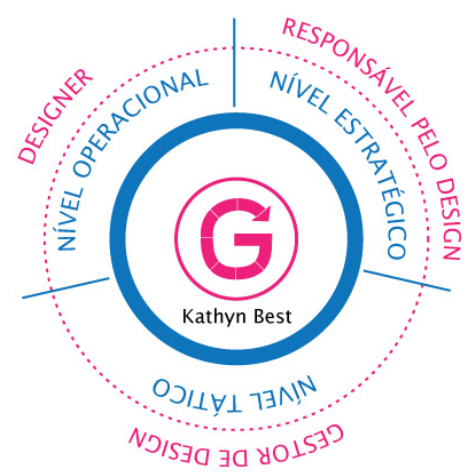

Fonte: Adaptado de Best (2009) e Mozota (2003)

A figura 2 mostra os componentes e os níveis da gestão de design no qual atuam. Estes são os componentes principais da gestão de design. Cada um possui papel de destaque nas atividades e em todo o fluxo de desenvolvimento das atividades.

A pesquisa realizada por Silva (2012), em que aplicou a abordagem sistêmica para a gestão de design sustentável, teve como um dos resultados o mapeamento sistêmico da gestão de design de um estúdio de design. No contexto apresentado pelo sistema da figura 3, Silva (2012) identificou os componentes da gestão de design apresentados por Mozota (2003) e Best (2009): responsável pelo design, gestor de design e o designer. Além destes, ela também encontrou outros componentes que, apesar de não executam os principais papéis, também estão presentes: administrativo/ financeiro, atendimento (comercial), produção e limpeza/ manutenção, figura 3. Além dos componentes, também identificou as interações existentes entre eles, mostrando as entradas e saídas de informações, material el ou energia. As interações do mapeamento da gestão de design de SILVA (2012) acontecem nos pontos de encontro entre os componentes do sistema e, com isso, pode-se identificar as tarefas desenvolvidas por cada um dos integrantes.

Compreender a gestão de design, suas interações e sistemática de funcionamento colaborou para o desenvolvimento da prática sistêmica da gestão de design e, consequentemente, para a obtenção de informações necessárias para o desenvolvimento de uma ferramenta de suporte à gestão de design.

\section{PROCEDIMENTOS METOLOLÓGICOS}


A pesquisa desenvolvida se caracteriza, conforme Gil (2010), por ser do tipo aplicada, que gera conhecimentos para aplicação prática e dirigidos à solução de problemas específicos. Tem abordagem qualitativa, com o objetivo de explorar o contexto da pesquisa composto pela gestão de design em um estúdio de design de pequeno porte localizado na cidade de Florianópolis. A pesquisa de objetivo exploratório visa obter maior familiaridade com 0 problema, explicitando-o. Ela busca reunir informações gerais sobre o objeto de estudo.

Quanto aos procedimentos técnicos, conforme Gil (2010), trata-se de um estudo de caso, envolvendo estudo aprofundado sobre a gestão de design do caso estudado, afim de conhecer o seu contexto, componentes e interações. O caso estudado é uma empresa ${ }^{1}$ de pequeno porte de design, localizada em Florianópolis, com aproximadamente dez colaboradores (o número sofreu variação ao longo do estudo).

Figura 3: Sistema detalhado da gestão de design da MPE

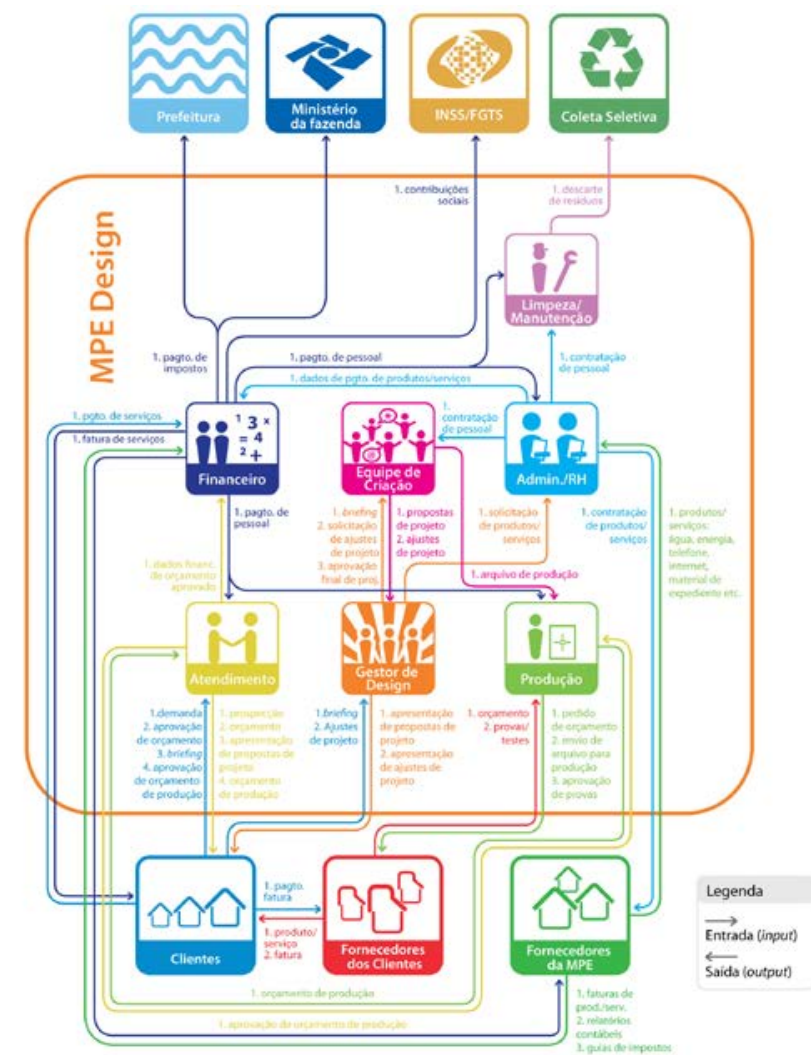

Fonte: SILVA (2012)

A pesquisa tomou como referência o mapeamento sistêmico desenvolvido por Silva (2012),

1

$\square$ Nuovo Design (www.nuovo.com.br) 
figura 3. Este mapeamento foi desenvolvido em estudos realizados no mesmo estúdio pesquisado e apresenta os componentes relacionados à gestão de design e suas interações, mostrando as entradas e saídas de informações, material e/ ou energia. A pesquisa realizada é uma continuidade à pesquisa desenvolvida por Silva, com foco na usabilidade de uma ferramenta de suporte à gestão de design.

A coletada de dados se deu por meio de observação participante entre os períodos de setembro a novembro de 2013 e, ao final, foram realizadas entrevistas semi-estruturadas com oito dos dez colaboradores do estúdio. As funções dos colaboradores entrevistados são diversas entre si (ver figura 3), como designer, gestor de design, administrativo/ financeiro e comercial (atendimento). As entrevistas tiveram o objetivo de conhecer as dificuldades individuais e coletivas dos principais integrantes da gestão de design no estúdio.

\section{MAPEAMENTO SISTÊMICO DA GESTÃO DE DESIGN: NECESSIDADES E DIFICULDADES}

Os resultados da pesquisa realizada foram obtidos por meio do uso do mapeamento sistêmico da gestão de design desenvolvido por Silva (2012) e por meio de estudo de caso, através da observação participante e da realização de entrevistas com os integrantes da gestão de design. O caso estudado é o mesmo estudado por Silva (2012) e trata-se de um estúdio de design de pequeno porte localizado em Florianópolis, que não faz uso de nenhuma ferramenta específica de gestão de projetos. A forma de organização da gestão de design do estúdio se dá por meio de algumas ferramentas de controle desarticuladas entre si, como agenda compartilhada, arquivos compartilhadas de pauta e briefing, agenda individual, caderno pessoal de anotações, documentos in loco de orçamentos etc.

Os principais componentes da gestão de design identificados por Silva (2012), entre os quais acontecem interações são: o responsável pelo design (no caso estudado, os clientes); o gestor de design; e o designer (no caso estudado, a equipe de criação). O responsável pelo design (clientes), está localizado fora da estrutura da empresa, por se tratar de um estúdio de design que presta serviços para terceiros. São eles que definem as estratégias em nível empresarial e dão a aprovação final em relação ao desenvolvimento dos projetos. No entanto, também foi verificado que existem outros componentes importantes como comercial (denominado como atendimento), produção, administrativo e financeiro.

As interações atribuídas aos outros componentes identificados na gestão (fornecedores, limpeza/ manutenção, INSS, Prefeitura etc.) ainda não serão consideradas neste trabalho, por ter sido identificado que as interações deles com a gestão de design sejam pequenas e não apresentem problemática suficiente para haver necessidade de comunicação com a ferramenta a ser desenvolvida. Um caso que será desconsiderado são as interações com fornecedores. Isso pelo pequeno porte do caso estudado, em que não há um volume de interações suficiente que justifique uma ferramenta automática de compras de produtos e serviços. No entanto, é um módulo a ser considerado na construção de uma ferramenta completa e adequada a todos os portes de entidades que farão uso da ferramenta. Tendo estas considerações em vista, uma síntese do mapeamento foi desenvolvida, priorizando a abrangência constatada no estudo de caso, o qual se apresenta na figura 4. 
Figura 4: Síntese do sistema da gestão de design da MPE

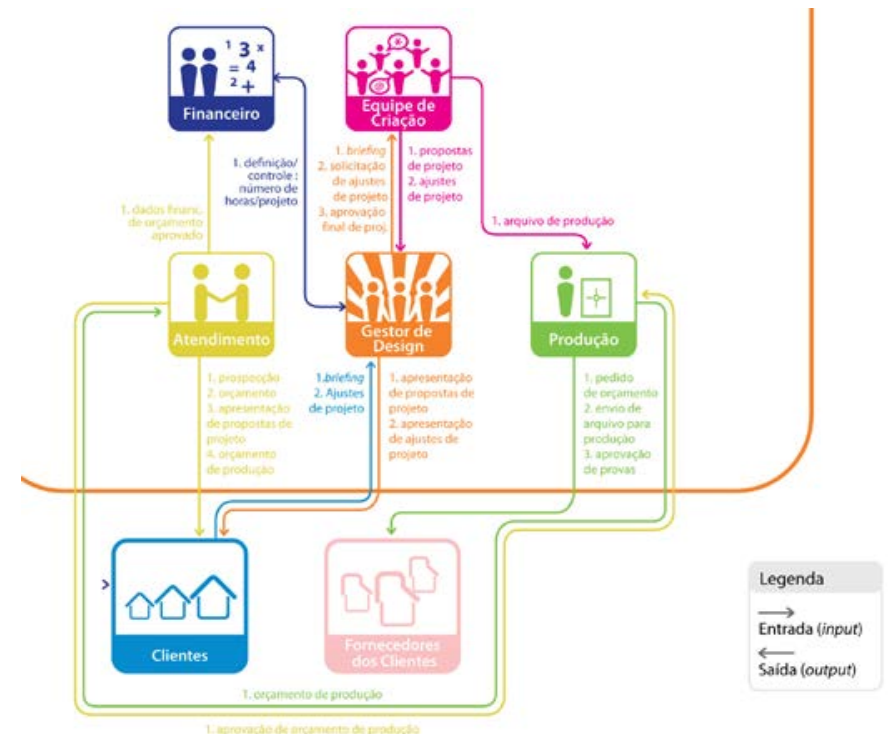

Fonte: Adaptada de SILVA (2012)

As principais dificuldades e necessidades de interação encontradas na gestão de design, que envolvem os componentes do sistema sintetizado, foram as seguintes:

- Necessidade de monitorar todas as etapas de projeto;

- Necessidade de facilitar o trabalho dos componentes envolvidos na gestão de design;

- Etapas de processo lentas;

- Necessidade de maior qualidade de processo;

- Necessidade de controle do tempo;

- Incidência de erros.

Estes necessidades e dificuldades são apresentadas a seguir, verificando quais os componentes envolvidos e analisando as principais interações entre eles que tem relação com as causas.

\subsection{Necessidade de monitorar todas as etapas de projeto}

A falta de uma ferramenta de gestão de design gera a dificuldade de controlar todas as etapas do projeto de design. A figura 4, com o sistema que a ferramenta terá abrangência, pode-se visualizar todos os componentes e as etapas que a ferramenta pode auxiliar na execução, monitorar ou seu desenvolvimento. A gestão de design integrada em uma só ferramenta pode facilitar o recebimento e a inserção de informações, além da visualização completa de todos as etapas de projeto, com possibilidade de visualizar os prazos, o andamento, o responsável em cada etapa etc. Além disso, pela falta ferramentas que proporcionem a documentação das 
atividades que são desenvolvidas, há uma dificuldade de visualizar como o processo de desenvolvimento ocorreu, por não haver o registro histórico das atividades.

\subsection{Necessidade de facilitar o trabalho dos componentes envolvidos na gestão de design}

Esta necessidade vem principalmente pelas falta de um local único para documentar todas as tarefas a serem executadas, bem como as informações, prazos e necessidades dos projetos. As atividades ficam desarticuladas pelo uso de ferramentas que não se conectam entre si, como documentos de briefing, agenda eletrônica, agenda pessoal ou caderno de anotações pessoal, documentos de orçamentos etc.

Figura 5: Interações onde há problema na troca de informações no processo de gestão de design

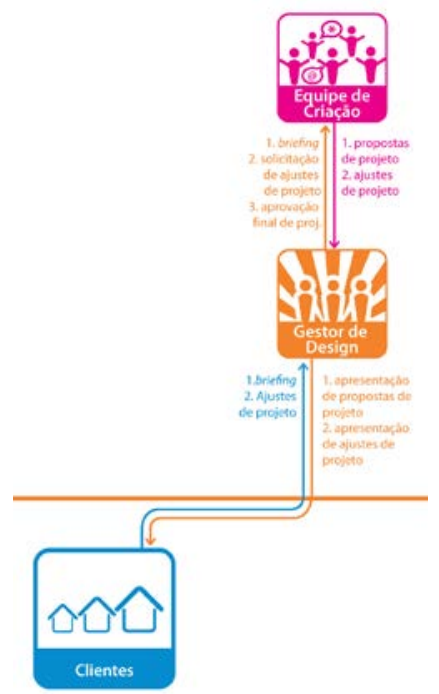

Fonte: Adaptado de SILVA (2012)
Figura 6: Interações que acontecem com problemas de lentidão na gestão de design

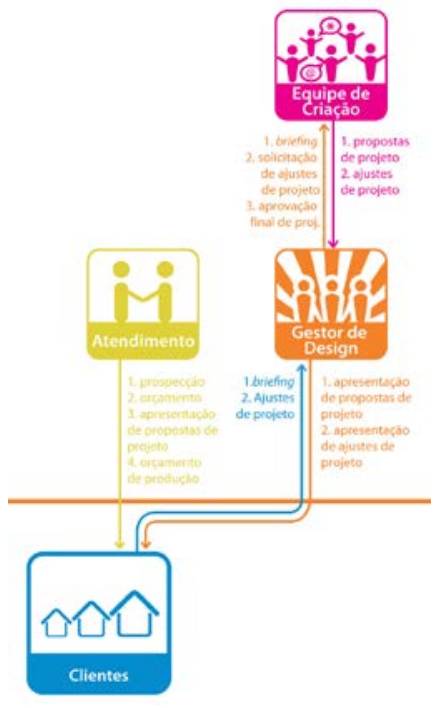

Fonte: Adaptado de SILVA (2012)

A figura 5 mostra as principais interações apresentam dificuldades em controlar as informações, que são principalmente entre o cliente e o gestor de design e entre o gestor de design e o designer. Os problemas desta dificuldades de interação, em geral, ficam evidentes na finalização do projeto, em que se constata que uma informação ficou faltando ou foi compreendida de forma equivocada, gerando falhas do produtos final. As interações mais problemáticas neste sentido acontecem na hora de receber ou passar o briefings e os ajustes de projeto, entre o cliente e o gestor de design e entre o gestor de design e o designer (equipe de criação).

\subsection{Etapas de processo lentas}

Praticamente todas etapas de passagem de informação dependem de reunião, o que fica também na dependência de disponibilidade de horário dos componentes envolvidos, deixando o processo lento. Em casos como pedidos de ajustes, ou acréscimo de informação em um mesmo briefing, ou mesmo definição de um prazo posterior à etapa de briefing não haveria 
necessidade de uma reunião, mesmo que rápida, entre as pessoas. A postagem das informações no sistema poderia ajudar a resolver este problema.

Na pesquisa, a principal causa de lentidão se encontra nas interações observadas na figura 6 , que acontece entre o atendimento e o cliente, na hora da elaboração dos descritivos para envio do orçamento ao cliente, entre gestor de design e o cliente, na hora de receber informações de briefing e ajustes e, por fim, entre gestor de design e o designer, na hora de passar as informações de ajustes, prazos e briefings. De forma geral, todos os integrantes também declararam haver lentidão no trabalho pela necessidade de alimentar diversas ferramentas (agenda pessoal, agenda eletrônica, documentos eletrônicos etc.) e pela necessidade de repetir algumas das informações em cada uma delas, por falta de integração das ferramentas utilizadas. Com estas etapas facilitadas pela ferramenta a ser desenvolvida, pode-se dedicar 0 tempo em atividades mais produtivas para a gestão de design.

\subsection{Necessidade de maior qualidade de processo}

A quantidade de informações que se perdem no processo, pela falta de um local para armazená-las e recuperá-las com facilidade, de forma acessível a todos os envolvidos nos projetos, bem como a quantidade de tempo que é destinada a resolver problemas por falta de interação e documentação por ser resolvida pela ferramenta a ser desenvolvida.

Figura 8: Componentes que necessitam de maior Figura 7: Interações entre Gestor de design controle do tempo em suas interações na gestão e Designers de design

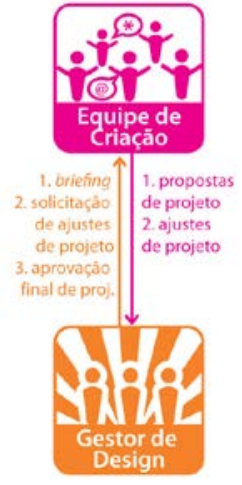

Fonte: Adaptado de SILVA (2012)

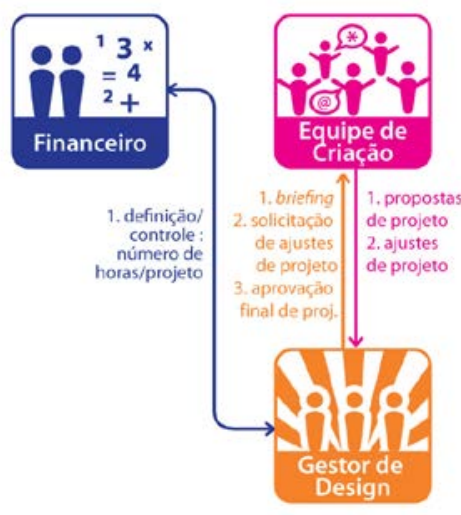

Fonte: Adaptado de SILVA (2012)

Além disso, um projeto mais organizado e claro permitirá o melhor uso do tempo, destinado-o para atividades de pesquisa e desenvolvimento de soluções de maior qualidade. Os gestores de design e os designers da equipe de criação são os mais afetados neste quesito. A figura 7 ilustra as interações/ atividades que, se a ferramenta ajudar a executar em um tempo menor, podem influenciar em um aumento da dedicação em outras atividades que podem trazer maior 
qualidade às soluções.

\subsection{Necessidade de controle do tempo}

O controle do tempo se faz necessário principalmente na etapa de execução do projeto pelo designer, desta forma é possível verificar se está correndo conforme orçamento aprovado junto ao cliente, calculando o custo em função do tempo destinado à execução do projeto.

$\mathrm{Na}$ figura 8 pode-se visualizar os componentes que tem maior necessidade do controle do tempo, tanto para visualização e acompanhamento do andamento e dos prazos (gestor de design), como para análise e controle das horas destinas ao projeto(aministrativo/ financeiro), e também para ajudar a gerenciar o tempo destinado às atividades, com lembretes e uma agenda conectada aos prazos dos projetos (designer).

\subsection{Incidência de erros}

Os erros acontecem, geralmente, por falta de documentação de informação ou de uma documentação sem um processo formalizado e adequado às atividades, conforme visto no item 5.2. Ela acontece principalmente no recebimento das informações de ajustes de projetos vindo do cliente e do gestor de design. Neste processo de armazenamento ou, na falta de armazenamento das informações recebidas, é que ocorre a maioria dos problemas que se tornam erros mais tarde, quando não são identificados antes da produção do produto. A identificação dos erros acontece nas provas de impressão, testes de produção ou quando 0 material chega produzido ao cliente, sendo tarde para possíveis correções.

De forma geral, as entrevistas com os integrantes do estúdio revelaram que eles sentem a necessidade de uma organização nas interações entre as partes do sistema formado pela gestão de design. Na visão deles, uma ferramenta para dar suporte às atividades de gestão de design deve controlar todas as etapas de projeto, deste a concepção de estratégias para desenvolvimento até suas etapas finais de entrega e retorno sobre a satisfação com o produto ou serviço executado. Que possa facilitar o trabalho dos componentes envolvidos na gestão de design, principalmente do gestor de design e do designer, que executam a maior parte das tarefas concernentes à gestão de design. E que possa como objetivo final se tornar mais eficaz (agilizar o processo), eficiente (trazer maior qualidade ao processo, menor incidência de erros etc.) e traga maior satisfação na execução das tarefas da gestão de design.

\section{CONSIDERAÇÕES FINAIS}

Esta foi uma pesquisa para levantar os principais componentes, interações, necessidades e dificuldades existentes nas interações da sistêmica de funcionamento da gestão de design afim ter, no futuro, fundamentação para estabelecer requisitos de usabilidade para 0 desenvolvimento de uma ferramenta destinada à gestão de design que posso colaborar com a solução de problemáticas encontradas. A abordagem sistêmica da gestão de design contribuiu para que fossem levantadas as informações necessárias sobre o contexto, os componentes e, principalmente, sobre as interações existentes. Como se tratou de um estudo de caso, aconselha-se que antes de partir para o desenvolvimento efetivo da ferramenta, seja interessante estudar outros casos em outros tipos de ambientes em que a ferramenta vai pode 
atuar. Por isso, a pesquisa objetiva ter continuidade para extrair mais informações que sejam relevantes para o desenvolvimento da ferramenta de gestão de design, pesquisando outros casos e contextos de uso, como departamentos de design dentro de organizações, núcleos de design etc.

Como a ferramenta ainda não foi concebida, ainda não se sabe qual o formato que ela assumirá no futuro. Por este motivo, evitou-se de falar ao longo do texto sobre que tipo de material, veículo, meio ou forma de materialização da ferramenta, deixando livre para que a criação não fique limitada aos meios existentes nos dias atuais, que podem ser diferentes dentro de alguns meses.

Uma ferramenta para dar apoio à gestão de design precisa atender às necessidades dos integrantes das atividades, que realizam tarefas específicas da área. A "usabilidade é uma consideração importante no projeto de produtos uma vez que ela se refere à medida da capacidade dos usuários em trabalhar de modo eficaz, efetivo e com satisfação." NBR ISO 9241-11 (2002:3). As interações entre as partes, como o tipo de informação e material trocados, são específicos do cada contexto, por este motivo é importante o estudo aprofundado sobre a atividade e interação existente na gestão de design, para que se possa desenvolver um produto com usabilidade.

\section{REFERÊNCIAS BIBLIOGRÁFICAS}

BERTALANFFY, L. V. Teoria geral dos sistemas. Petrópolis: Vozes, 2008.

BEST, K. Le design management: stratégie, méthode et mise en ouvre. Paris: Pyramide, 2009.

CAPRA, Fritjof. O ponto de mutação. 15. ed. São Paulo: Cultrix, 1993. 447p

CYBIS, W.; BETIOL, A. H.; FAUST, R. Ergonomia e usabilidade: conhecimentos, métodos e aplicações. 2.ed. São Paulo: Novatec Editora, 2010.

GIL, A. C. Como elaborar projetos de pesquisa. São Paulo: Atlas, 2010.

GIMENO, J. M. I. La gestión del diseño en la empresa. Madrid: McGraw-Hill, 2000.

NBR 9241-11. Requisitos ergonômicos para trabalho de escritórios com computadores: orientações sobre usabilidade. Rio de Janeiro: ABNT, 2002.

JORDAN, P. W. An Introduction to Usability. Londres: Taylor \& Francis Ltda., 1998.

MOZOTA, B. B. Design management: using design to build brand value and corporate innovation. New York, 2003.

USABILITY PROFESSIONAL'S ASSOCIATION. What's usability? Disponível em:

$<$ http://www.upassoc.org/usability_resources/about_usability/index.html >. Acesso em: 11 de novembro de 2013.

VASCONCELOS, M. J. E. Pensamento sistêmico: o novo paradigma da ciência. 7. ed. Campinas:

Editora Papirus, 2002. 286 p.

SILVA, C. S. Abordagem sistêmica com foco na gestão de design sustentável [dissertação]: o caso Nuovo Design. Orientador, Luiz Fernando Gonçalves de Figueiredo. Florianópolis, SC, 2012. 